\title{
Field line resonances in discretized magnetospheric models: an artifact study
}

\author{
M. Stellmacher ${ }^{1,2}$, K.-H. Glassmeier ${ }^{2}$, R. L. Lysak ${ }^{3}$, M. G. Kivelson ${ }^{1}$ \\ ${ }^{1}$ Institute of Geophysics and Planetary Physics, University of California at Los Angeles, California, USA \\ 2 Institut für Geophysik und Meteorologie, Technische Universität Braunschweig, Germany \\ ${ }^{3}$ School of Physics and Astronomy, University of Minnesota, Minneapolis, USA
}

Received: 3 May 1996 / Revised: 6 January 1997 / Accepted: 22 January 1997

\begin{abstract}
For more than two decades numerical models of the Earth's magnetosphere have been used successfully to study magnetospheric dynamic features such as the excitation of ULF pulsations and the mechanism of field line resonance. However, numerical formulations simplify important properties of the real system. For instance the Alfvén continuum becomes discrete because of a finite grid size. This discretization can be a possible source of numerical artifacts. Therefore a careful interpretation of any observed features is required. Examples of such artifacts are presented using results from a three dimensional dipole model of the magnetosphere, including an inhomogeneous distribution of the Alfvén velocity.
\end{abstract}

\section{Introduction}

The Earth's magnetospheric system cannot be described by analytical models in all of its complexity. Simplifications are necessary even for the solutions of very specific problems. Nevertheless, early analytical studies have led to improved understanding of the basic physics of many magnetospheric processes.

A particularly well-studied aspect of magnetospheric physics is the theory of magnetohydrodynamic (MHD) waves. This commonly accepted theory predicts that the cold magnetized plasma of the outer magnetosphere supports two different wave modes. One of these wave modes is the isotropic and non-localized compressional mode, also referred to as the fast mode or poloidal mode. The other one is the localized toroidal mode, also called the Alfvén mode that propagates exclusively along the field lines. These two modes always couple in a non-uniform plasma if any wave disturbances are not fully axisymmetric. Therefore, no pure modes exist in realistic situations, and the wave systems are complex with transverse and compressional contributions.

Correspondence to: M. Stellmacher
Many analytical studies treat the magnetosphere as a box with a straight magnetic field topology and ideal boundary conditions (Radoski, 1971). Because the two wave modes remain coupled as long as one assumes nonuniformity of the background conditions, these studies have been useful in developing a number of theories, such as the origin of ultralow frequency (ULF) pulsations (Dungey 1954; Allan and Poulter, 1992, and references therein), models of instabilities at the magnetopause as a possible source of MHD wave excitation (Parker, 1958; Southwood, 1968), the cavity mode as a possible explanation for the observed discrete spectrum of the compressional mode (Kivelson et al., 1984; Kivelson and Southwood, 1985, 1986; Allan et al., 1986) and the mechanism of field line resonance (FLR) as a result of the coupling between the discrete compressional mode and the Alfvén continuum (Southwood, 1974; Chen and Hasegawa, 1974a, b; Radoski, 1976). Only a small number of analytical approaches have been published, taking into account a curvature of the ambient magnetic field (Hameiri, 1985; Inhester, 1986; Wright and Thompson, 1994; Lifshitz, 1987; Kouznetsov and Lotko, 1995).

The need to understand wave properties in more realistic systems led to the exploitation of numerical models. The simplified box model approximation was adequate for investigation of further properties of the MHD wave modes, such as temporal development and phase mixing (Pritchett and Dawson, 1978; Inhester, 1987; Southwood and Kivelson, 1990; Mann et al., 1995). More sophisticated models have included the curvature of magnetic field lines, as for example in the cylindrical model (Radoski, 1974; Allan et al., 1985, 1986; Allan and Mc Diarmid, 1993), the wave guide model (Rankin et al., 1993; Wright, 1994), and the dipole model (Lee and Lysak, 1989, 1991a). The latter is used for the simulations presented in this study.

Although numerical models have improved the understanding of magnetospheric features, they still neglect important properties of the real system, such as asymmetry of the global structure (the magnetospheric 
tail is ignored) and proper boundary conditions. The consequences of using a discretized representation of the Alfvén continuum because of finite grid sizes in numerical models have been studied in much detail (Appert et al., 1975; Pritchett and Dawson, 1978; Appert et al., 1984; Lee and Roberts, 1986). The discretized systems are found to behave like a finite number of independent oscillators instead of a real continuum, and this modifies the physics.

Rickard and Wright (1994) have investigated the relevance of phase mixing in a numerical box model that includes finite grid spacing. They came to the conclusion that this process leads to a limit on the time one can run a simulation correctly. This time can be governed by the phase mixing length, defined as the length over which the phase of neighboring Alfvén waves differ by $2 \pi$, which is found to decrease with time. The resolution of this length in the numerical model is one requirement for an accurate simulation.

This study introduces examples of artifacts caused by the discretization of the Alfvén continuum. These artifacts lead to the formulation of a critical time limit to be used for the simulations, similar to that introduced by Rickard and Wright (1994). This work investigates in detail the effects of the discretization when the simulation approaches and exceeds this limit.

In a recent study Ding et al. (1995) examined the excitation of the poloidal mode field line resonance using a two dimensional ideal MHD model. In their results they find a slow amplitude modulation in the transverse components that is caused by beating of two toroidal oscillations; the modulation period depends on the number of grid points used in the radial direction. They acknowledge the fact that, because of the discrete numerical model, the eigenfunctions associated with the localized poloidal resonance collapse to grid scale singular functions. They conclude that ideal MHD theory is inadequate for this kind of study. We do not fully agree with this general statement as the results presented in this work indicate that discrete models provide reasonable results as long as a certain critical time limit, which is controlled by the radial grid spacing, is not exceeded. Mann and Wright (1995) came to a similar conclusion concerning the study by Ding et al. (1995) when they discussed the problem of phase mixing in numerical models.

The study is structured as follows: the following sections review briefly the analytical approach to the coupled MHD mode problem and describe how the equations are modified for use in numerical approaches; the next sections introduce some possible consequences of the numerical formulation and analyzes artifacts that arose during runs of the three dimensional dipole model; finally the validity of discretized magnetospheric models in studying ULF pulsations and related phenomena is assessed.

\section{General analytical approach}

Most analytical studies have adopted Radoski's (1971) box model. This model considers a cold plasma contained in a straight uniform magnetic field $\vec{B}_{0}=B_{0} \hat{z}$. The plasma density $\rho_{0}(x)$ is chosen to be a function of $x$, the radial direction across the magnetic shells, in order to retain the coupling of wave modes. The boundary conditions in the $x$ and $z$ direction are often assumed to be perfectly reflecting. The finite length of the field lines requires quantization of the wave number $k_{z}$. No boundaries are assumed in the azimuthal (y) direction, but the waves have finite azimuthal wave numbers.

Linearized MHD perturbations in a cold plasma satisfy

$$
\begin{aligned}
& \nabla \times \vec{b}=\mu_{0} \vec{j} \\
& \nabla \times\left(\vec{u} \times \vec{B}_{0}\right)=\frac{\partial \vec{b}}{\partial t} \\
& \rho_{0} \frac{\partial \vec{u}}{\partial t}=\vec{j} \times \vec{B}_{0} \\
& \vec{E}=-\vec{u} \times \vec{B}_{0},
\end{aligned}
$$

where $\vec{B}_{0}$ and $\rho_{0}$ are the background magnetic field and density and $\vec{j}, \vec{u}, \vec{b}$ and $\vec{E}$ are the perturbations of the magnetospheric currents, plasma velocity, magnetic field and electric field, respectively.

By elimination, the following expressions are obtained:

$$
\begin{aligned}
& \left(\frac{1}{v_{A}^{2}} \frac{\partial^{2}}{\partial t^{2}}-\frac{\partial^{2}}{\partial x^{2}}-\frac{\partial^{2}}{\partial z^{2}}\right) E_{y}=-\frac{\partial^{2} E_{x}}{\partial x \partial y} \\
& \left(\frac{1}{v_{A}^{2}} \frac{\partial^{2}}{\partial t^{2}}-\frac{\partial^{2}}{\partial y^{2}}-\frac{\partial^{2}}{\partial z^{2}}\right) E_{x}=-\frac{\partial^{2} E_{y}}{\partial x \partial y},
\end{aligned}
$$

$v_{A}=B_{0} / \sqrt{\mu_{0} \rho_{0}}$ being the Alfvén velocity, $E_{y}$ corresponding to the poloidal mode and $E_{x}$ to the toroidal mode.

One possible way to treat these equations is to employ the Laplace transform on the time variable to solve the corresponding initial value problem (Sedlacek, 1971; Zhu and Kivelson, 1988). The Green function of the differential equation is constructed, and slowly decaying quasi-eigenmodes of the system can be inferred from its singularities.

Another way to deal with the equations is mathematically simpler and has frequently been used to determine the quasi-eigenmodes (Barston, 1964; Southwood, 1974; Nishida, 1978). The electric field vector can be written as

$\vec{E}=\left(E_{x}(x), E_{y}(x), 0\right) \exp \left[i\left(k_{y} y-k_{z} z-\omega t\right)\right]$,

with the field aligned/azimuthal wavenumber $k_{z} / k_{y}$. Equations (5) and (6) become

$\left(\frac{\omega^{2}}{v_{A}^{2}}+\frac{d^{2}}{d x^{2}}-k_{z}^{2}\right) E_{y}=i k_{y} \frac{d}{d x} E_{x}$

$\left(\frac{\omega^{2}}{v_{A}^{2}}-k_{y}^{2}-k_{z}^{2}\right) E_{x}=i k_{y} \frac{d}{d x} E_{y}$. 
Note that for axisymmetric disturbances $\left(k_{y}=0\right)$, the equations for $E_{x}$ and $E_{y}$ decouple. The solution for the poloidal mode $\left(E_{y}\right)$ satisfies

$$
\begin{aligned}
\frac{d^{2} E_{y}}{d x^{2}}-k_{y}^{2} \frac{d M^{2}}{d x} & \frac{1}{\left(M^{2}-k_{z}^{2}\right)\left(M^{2}-k_{y}^{2}-k_{z}^{2}\right)} \frac{d E_{y}}{d x} \\
& +\left(M^{2}-k_{y}^{2}-k_{z}^{2}\right) E_{y}=0,
\end{aligned}
$$

where $M^{2}(x)=\omega^{2} / v_{A}^{2}(x)$ is a function of $x$ (Southwood, 1974).

Assuming the density to vary monotonically with $x$, this equation has two singularities of physical significance. Where $\left(M^{2}-k_{y}^{2}-k_{z}^{2}\right)=0$ the solutions for $E_{y}$ change from being oscillatory to decaying exponentially with distance. This point $x_{t}$ is known as the turning point of the poloidal mode. Where $\left(M^{2}-k_{z}^{2}\right)=0\left(x_{r}\right)$, the perturbations are found to be in resonance with the transverse mode $\left(E_{x}\right)$. This is where the field line resonances occur. A nonmonotonic function for the density profile might result in a larger number of singularities and more turning points and/or resonance points.

Solutions of Eq. (10) can be found in the vicinity of the singularities. Near the turning point $x_{t}$ there are two independent solutions of the form

$u_{1}=a_{0}+\sum_{n=1}^{\infty} a_{n}\left(x-x_{t}\right)^{n}$

$u_{2}=\left(x-x_{t}\right)^{2}\left\{b_{0}+\sum_{n=1}^{\infty} b_{n}\left(x-x_{t}\right)^{3 n}\right\}$,

which are both finite at $x_{t}$.

In the vicinity of the resonance $x_{r}$ a solution can be found in the form of a superposition of modified Bessel functions $I_{0}$ and $K_{0}$ of order zero (Southwood, 1974)

$E_{y}=A I_{0}\left(k_{y}\left(x-x_{r}\right)\right)+B K_{0}\left(k_{y}\left(x-x_{r}\right)\right)$.

However, without further treatment the solutions become logarithmically infinite at the singularity. This causes mathematical problems and is physically not satisfying. Obviously the ideal MHD equations are not valid around this singularity. The most commonly used resolution is to introduce a complex frequency $\omega=\omega_{r}+i \omega_{i}$, with a small positive imaginary part $\omega_{i}$ (Southwood, 1974; Bertin et al., 1986). This can be thought of as describing dissipation effects that prevent an infinite increase of the amplitude at the resonance point. At the same time, dissipation also causes a broadening of the resonance width. Dissipation effects are equivalent to sinks of electromagnetic energy, which can, for example, be caused by a permeable magnetopause or plasmapause, the resistive ionosphere or by collisionless damping in the real magnetosphere. Energy considerations show that normal mode solutions, even in a simplified system like the one used here, are not possible for $\omega_{i} \neq 0$. The limit where $\omega_{i} \rightarrow 0$, however, is mathematically allowed. The poloidal mode still damps, for example through phase mixing.

The solutions described have been called quasieigenmodes. They decouple in certain limits $(m=0$ or $m \rightarrow \infty, m$ being the azimuthal wave number of the perturbations) and become true eigenmodes, but they are generally coupled. However, theoretical studies have shown that this coupling is a one-way process. An energy source, usually of compressional nature due to instabilities at the magnetopause or pressure pulses in the solar wind, excites a discrete poloidal mode spectrum. These compressional waves, which are oscillatory outside their turning points and decaying within, are being damped with time, even in the absence of dissipation, through the coupling to the toroidal mode (Radoski, 1974; Appert et al., 1984; Kivelson and Southwood, 1985). They excite the Alfvén continuum (Bertin et al., 1986). FLRs now occur, where the frequency of the poloidal mode matches an eigenfrequency of the field lines. Previous theoretical work has shown that at the limit of long times the energy of the compressional oscillations couples irreversibly into the excited FLRs (Radoski, 1974, 1976; Lee and Roberts, 1986; Wright, 1994). In the absence of dissipation mechanisms, the energy is trapped in the toroidal mode.

\section{Numerical approach}

The introduction of dipole magnetic geometry greatly complicates the analysis of MHD wave properties. Even early work (Cummings et al., 1969) required numerical solutions to identify wave structure and resonant frequencies. More recently, a number of different numerical models of waves in a dipole magnetosphere have been investigated, but most of them use basically the same simplifications and techniques. The model used for the simulations presented in this study includes a three dimensional dipole magnetic field as well as inhomogeneous plasma conditions. It was first introduced by Lee and Lysak (1989) as a two dimensional dipole model. Later Lee and Lysak (1991a) published the first results of the extended three dimensional magnetosphere. Figure 1 shows a meridional plane with the dipole coordinates $(\hat{\mu}, \hat{v}, \hat{\phi})$, where $\hat{\mu}$ is directed along the field lines, $\hat{v}$ is normal to the field lines pointing outward and $\hat{\phi}$ is the usual azimuthal direction $(\hat{\mu}=\hat{v} \times \hat{\phi})$.

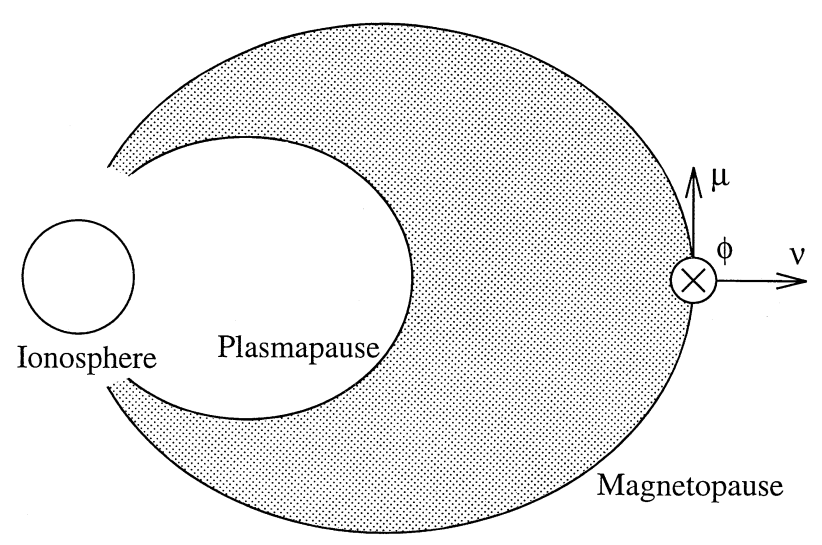

Fig. 1. The dipole magnetic field geometry with the coordinate system and the boundaries used in this model 
The boundaries of the model are the plasmapause at $L=5$ ( $L$ is the Mc Ilwain parameter), the magnetopause at $L=10$ and the ionosphere at constant $\mu$ at approximately 1 Earth radius. All boundaries are considered as perfect reflectors due to large Alfvén velocity gradients and perfect conductivity, respectively. The density $\rho_{0}$ and the ambient magnetic field $\vec{B}_{0}$ are inhomogeneous in the meridional plane, but symmetric in the azimuthal direction. This model evidently includes field line curvature, varying field line length and inhomogeneity of both the magnetic field $\vec{B}_{0}$ and the density $\rho_{0}$.

The currents perpendicular to the background magnetic field can be written in the form:

$$
\begin{aligned}
& \rho_{0} \frac{\partial \vec{u}}{\partial t}=\vec{j} \times \vec{B}_{0} \\
& \Rightarrow \rho_{0} \frac{\partial\left(\vec{u} \times \vec{B}_{0}\right)}{\partial t}=\left(\vec{j} \times \vec{B}_{0}\right) \times \vec{B}_{0} \\
& \Rightarrow \vec{j}_{\perp}=\frac{1}{v_{A}^{2} \mu_{0}} \frac{\partial \vec{E}_{\perp}}{\partial t} .
\end{aligned}
$$

The differential equations solved in the model are derived from the linearized MHD- and Maxwell-equations for the cold plasma of the outer magnetosphere. The currents in Ampere's law are replaced by Eq. (16).

$$
\begin{aligned}
& \nabla \times \vec{E}=-\frac{\partial \vec{b}}{\partial t} \\
& \nabla \times\left.\vec{b}\right|_{\perp}=\frac{1}{v_{A}^{2}} \frac{\partial \vec{E}_{\perp}}{\partial t}
\end{aligned}
$$

A field aligned electric field component is neglected by assuming infinite conductivity of the plasma. In dipole coordinates, Eqs. $(17,18)$ may be written as

$$
\begin{gathered}
\frac{\partial b_{\mu}^{*}}{\partial t}=-\frac{h_{\mu}}{h_{v} h_{\phi}}\left(\frac{\partial E_{\phi}^{*}}{\partial v}-\frac{\partial E_{v}^{*}}{\partial \phi}\right) \\
\frac{\partial b_{v}^{*}}{\partial t}=\frac{h_{v}}{h_{\mu} h_{\phi}} \frac{\partial E_{\phi}^{*}}{\partial \mu} \\
\frac{\partial b_{\phi}^{*}}{\partial t}=-\frac{h_{\phi}}{h_{v} h_{\mu}} \frac{\partial E_{v}^{*}}{\partial \mu} \\
\frac{1}{v_{A}^{2}} \frac{\partial E_{v}^{*}}{\partial t}=-\frac{h_{v}}{h_{\mu} h_{\phi}}\left(\frac{\partial b_{\phi}^{*}}{\partial \mu}-\frac{\partial b_{\mu}^{*}}{\partial \phi}\right) \\
\frac{1}{v_{A}^{2}} \frac{\partial E_{\phi}^{*}}{\partial t}=\frac{h_{\phi}}{h_{v} h_{\mu}}\left(\frac{\partial b_{v}^{*}}{\partial \mu}-\frac{\partial b_{\mu}^{*}}{\partial v}\right),
\end{gathered}
$$

where $h_{\mu}=r^{3} / \sqrt{1+3 \sin ^{2} \Theta}, h_{v}=r^{2} / \cos \Theta \sqrt{1+3 \sin ^{2} \Theta}$ and $h_{\phi}=r \cos \Theta$ are the metric coefficients of the dipole coordinate system and $E_{i}^{*}=h_{i} E_{i}$ and $b_{i}^{*}=h_{i} b_{i}$. For the numerical solution of these differential equations, describing an initial-value problem, a modified staggered leapfrog scheme is used, which gives a second order accuracy in space and time. A more detailed discussion of the numerical model is given by Lee and

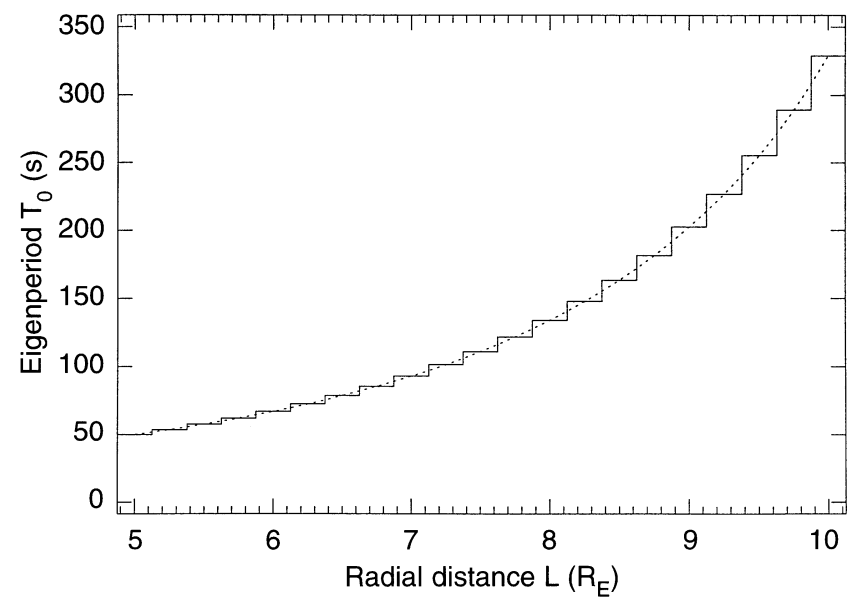

Fig. 2. The fundamental eigenperiod of the field lines in a continuum (dotted line) and in a discretized model plotted versus the equatorial distance to the flux tube in $R_{E}$

Lysak (1989, 1990, 1991a, b) and Lysak and Lee (1992).

The Alfvén continuum, which is the continuous spectrum of the toroidal mode, is produced by the change of the length of the field lines as well as the continuous variation of the Alfvén velocity in the radial direction. Because of a finite grid size in numerical models, the continuously changing eigenperiod of the field lines turns into a step function, as illustrated in Fig. 2. The dotted line shows the continuous function of the analytical approach, whereas the solid line shows the step function of the numerical model. This discretization in numerical models affects the resolution of the singularities in the system. Resonance-like structures turn out to be nearby field lines (grid points), excited through the finite bandwidth of the compressional mode. The exact position of the resonance cannot be determined numerically, it lies somewhere between the two grid points with the most singular behavior.

The solutions found in the discretized numerical system are 'real' eigenmodes, in contrast to the quasieigenmodes found in analytical systems. The 'real' eigenmodes couple in a time dependent system and decouple for $m=0 \quad(\partial / \partial \phi=0)$. However, the coupling appears to be somewhat different from the one obtained in the analytical approach. The continuum in the numerical models is represented by a system of single oscillators. The coupling in such a system is reversible, hence the energy cannot be trapped in the Alfvén continuum.

\section{Consequences of the discretization}

For generating wave disturbances, a compressional impulse $\left(E_{\phi}\right)$ of the form

$E_{\phi, 0}=A_{0} \sin \left(\omega_{0} t\right) \cos (m \phi) \exp \left(-A_{\mu} \mu^{2}-A_{\phi} \phi^{2}-A_{t} t\right)$,

is applied at the magnetopause. Here $\omega_{0}=2 \pi / T_{0}$ is the frequency of the impulse, $m$ is the azimuthal wavenum- 
ber and $A_{0}, A_{t}, A_{\mu}, A_{\phi}$ are constants. For the simulations in this study $T_{0}=50 \mathrm{~s}$ and $m=3$ was chosen. The source is localized around the equatorial plane, but global in the azimuthal direction, which prevents side effects due to an imhomogeneous azimuthal propagation of the waves. The impulse decays through $\exp \left(-A_{t} t\right)$ and is negligible after approximately 12 cycles of the wave $(600 \mathrm{~s})$.

The resolution of the grid used in the dipole model is sixty-four grid points along the field lines, 64 grid points in the azimuthal direction and either 41 or 82 grid points in the radial direction across the magnetic shells. This last resolution determines the discretization of the Alfvén continuum and changes the difference in the eigenfrequencies $\Delta f$ between two radially neighboring field lines.

The energy density (energy per unit $L$ ) of poloidal and toroidal modes versus the radial distance is shown in Fig. 3 for both grid resolutions. The temporal mean is taken over the total simulation time $(1 \mathrm{~h})$. The kinetic energy density at each time step is obtained from

$E_{\text {tor }}=\frac{1}{2 \mu_{0}} \iint\left(\frac{E_{v}^{2}}{v_{A}^{2}}+b_{\phi}^{2}\right) A(\mu, v) d \mu d \phi$

$E_{p o l}=\frac{1}{2 \mu_{0}} \iint\left(\frac{E_{\phi}^{2}}{v_{A}^{2}}+b_{\mu}^{2}+b_{v}^{2}\right) A(\mu, v) d \mu d \phi$.

$A(\mu, v)$ includes geometric factors and the metric coefficients. The electric energy of the modes $\left(E_{e l} \propto E^{2}\left(v_{A} / c\right)^{2}\right)$ is negligible in this system. It should be emphasized that as the two modes always couple the classification of the wave modes by their dominant field components is an approximation.

It is clear that as the resolution improves, more resonances can be resolved and the Alfvén continuum shows a more 'singular' behavior. The amplitudes of the resonances increase as their widths decrease. The

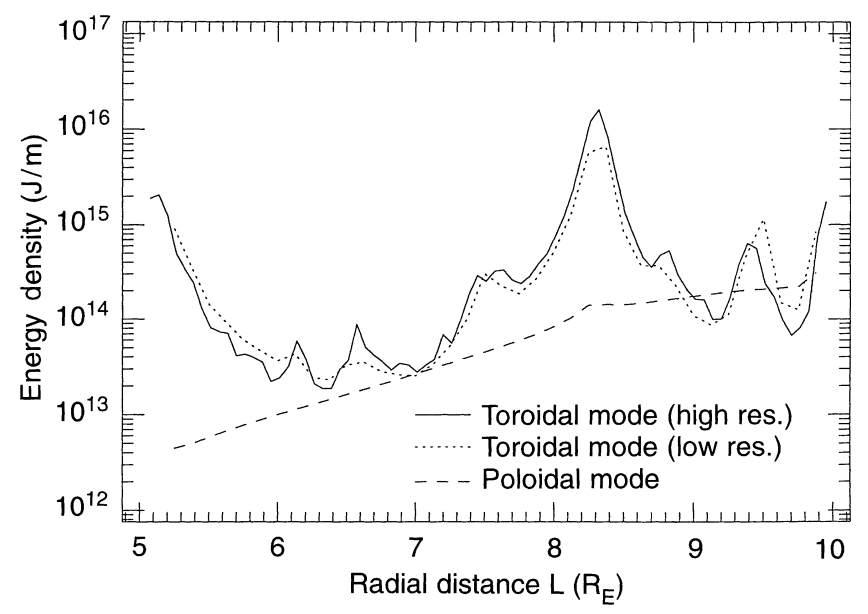

Fig. 3. The energy density profile of the toroidal mode for the high resolution grid (solid line), the low resolution grid (dotted line) and the poloidal mode (dashed line) over the radial distance, integrated over each magnetic shell and averaged over the total simulation time. Note the logarithmic scale envelope of the poloidal mode on the other hand does not change significantly with improved spatial resolution.

The spatial resolution of the Alfvén continuum depends on the radial grid size. Therefore the results shown in Fig. 3 are not surprising. The time dependence of the solution on the other hand, exhibits unexpected features. Figure 4 displays the energy density of both wave modes as well as their sum (the total energy of the system), now integrated over the whole magnetosphere as a function of time. Forty-one grid points were used across the magnetic shells in the radial direction for Fig. $4 \mathrm{a}$ and 82 for Fig. 4b.

The total energy in the model is much higher in the high resolution model, because the finer grid allows shorter wavelengths to be excited, whereas these cannot be represented on the larger grid. The numerical scheme produces no amplitude dissipation, therefore no wavelengths of any spectral band are being damped. Because the initial energy source used for these simulations allows a very broad frequency band to be excited, there is considerably more energy in the higher resolution case. Therefore a direct comparison of the magnitudes of the total energy in the model is not sensible and results such as amplitudes or energy density cannot be interpreted quantitatively.
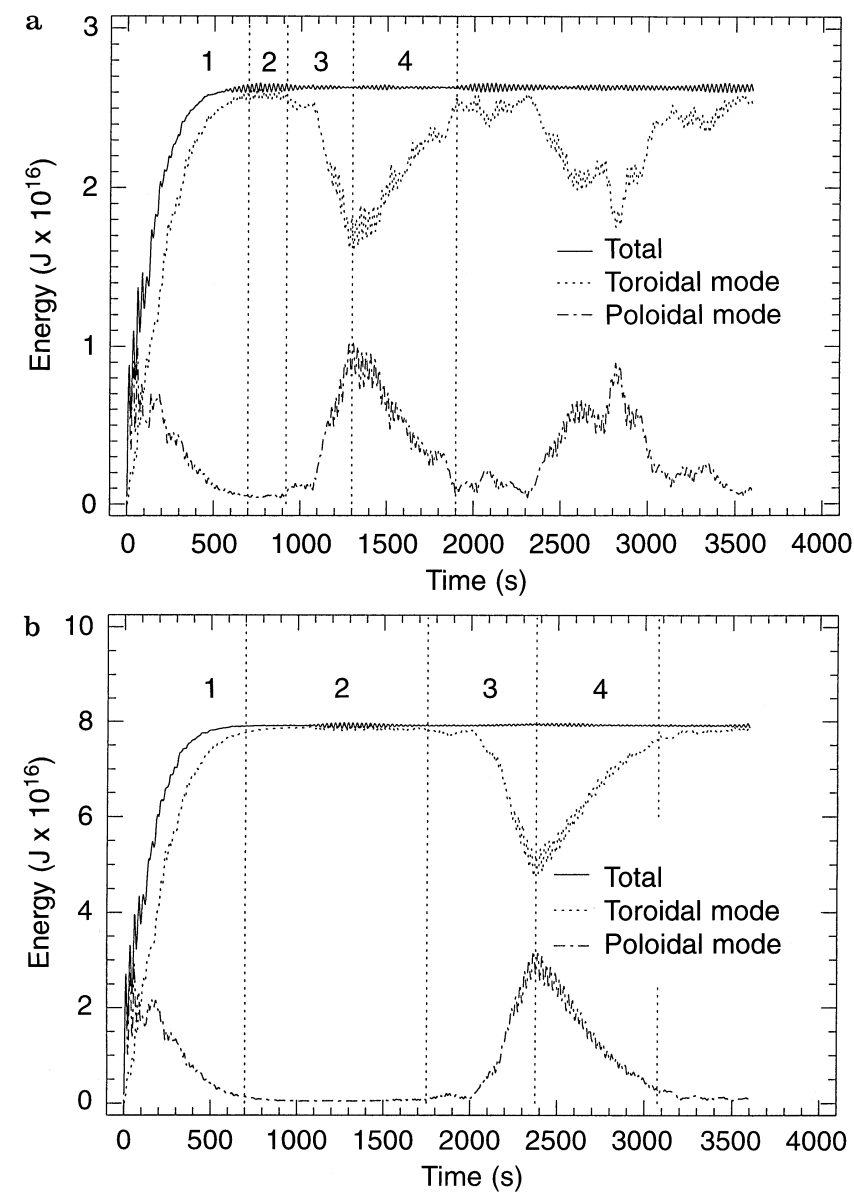

Fig. 4a, b. The temporal development of the total energy of the system (solid line) and the energy of the toroidal mode (dotted line)/poloidal mode (dotted-dashed line) in the a low resolution grid and $\mathbf{b}$ the high resolution grid 
It should be noted that the divergence of the magnetic field is of the order of $10^{-15}$ relative to the magnitude of the field and a typical scale length for both grid resolutions. This also indicates a good stability of the numerical scheme, similar for both resolutions.

The evolution of the energy can be separated into four phases (see vertical lines in Fig. 4). In phase 1 the compressional mode couples all of its energy almost immediately into the toroidal mode and thus into the field line resonance, as expected according to theoretical studies (e.g., Radoski, 1976; Wright 1994). In phase 2 the FLR is established and there is no more compressional energy available in the system. During phase 3 the FLR couples its energy back into the poloidal mode. This energy conversion reaches a maximum and recurs periodically. In phase 4 the poloidal mode has gained enough energy to excite toroidal modes anew.

From the energy density profile in Fig. 3 it is known that most of the toroidal energy lies in the magnetic shells next to the FLR at $L=8.3$. Thus these shells are expected to be mainly responsible for the signatures in the temporal development of the toroidal energy. Investigations of spatial energy distributions (not displayed here) confirm this. Only the magnetic shells near $L=8.3$ lose energy to the global compressional mode at the observed time.

How does the variation of the grid size affect the time scales of these phases? Phase 1 remains unchanged. The dominant mode in this phase is the global compressional mode, which does not depend on the grid size in the radial direction. The time scale of phase 2 more than doubles when halving the grid size. Phase 3 is approximately the same and phase 4 remains unchanged.

The physical parameter that changes with the grid size and that can be related to the wave modes is the difference of the eigenfrequencies $\Delta f$ of the radially neighboring field lines. On the two shells across the FLR at $L=8.3$ mainly the third harmonic modes are present. For the case of Fig. 4a (41 grid points) this implies $\Delta f=$ $0.76 \mathrm{mHz}$. Assuming a direct interaction between the azimuthal perturbations on two adjacent field lines, or more generally speaking between two oscillators in a coupled system, an empirical estimation of the cycle time of the mode conversion (onset of phase 4) is given by:

$T_{\text {cycle }}=\left|\left(f_{2}-f_{1}\right)\right|^{-1}=\left|\Delta f_{12}\right|^{-1}$,

where $f_{1}$ and $f_{2}$ are the frequencies of the perturbations or the oscillators. The cycle time is equivalent to the time the two waves need to go through one phase cycle (starting in phase, going out of phase, ending in phase), and is therefore related to the time-variation of the phase difference $\Delta \Phi$ between the perturbations on the two field lines. This calculation is consistent with the estimation of the cycle time in Fig. 4a, where the maximum of the mode conversion is reached after $\sim 1300$ s. For the case of Fig. $4 \mathrm{~b}$ ( 82 grid points), the cycle time is estimated as $\sim 2375 \mathrm{~s}$, and $\Delta f=0.42 \mathrm{mHz}$ at that particular resonant position in the model. Note that it is not necessarily the fundamental mode of the field lines that determines the cycle time, but the frequencies of the excited harmonics.

This empirical result is similar to the critical time theoretically governed by the idea of resolving the phase mixing length proposed by Wright (1994) and Mann et al. (1995). Rickard and Wright (1994) considered the resolution problem in an open cavity/wave guide in terms of the phase mixing length $L_{p h}$ explicitly and gave the criterion:

$d x<L_{p h}=2 \pi /\left(t d \omega_{A} / d x\right) \Longrightarrow t<\left|\Delta f_{12}\right|^{-1}=t_{p h}$,

where $d x$ is the grid spacing in the radial direction and $\omega_{A}$ is the Alfvén frequency of the field line. The time limit $t_{p h}$ in which simulations can be run accurately is now reached when the phase mixing length equals the grid spacing. A detailed explanation of the physical effect when the simulation approaches and exceeds this limit will be given in the following paragraphs.

To investigate the cause of this energy conversion in more detail, the magnetic field components were tracked at locations near the equator on the two magnetic shells closest to the FLR at $L=8.3$, using the higher resolution grid. The data was Fourier analyzed $(\Delta t=3 \mathrm{~s}, n=64$, shift $=16)$ and selected for the frequency interval around $20 \mathrm{mHz}$, which contains the third harmonic of the eigenfrequency of that particular field line. Figure 5 displays amplitude and phase of the perturbations on the shell with resonant frequency closest to the driving frequency. Phases are shown relative to a specifically chosen reference signal, of all three magnetic components versus time. It is not necessary to take the electric field components into account, because $E_{v}$ exhibits a behavior qualitatively identical to $b_{\phi}$, as well as $E_{\phi}$ to $b_{v}$. The phase is plotted only at times when the amplitude exceeds a specified minimum value.

The amplitudes of the magnetic field components evolve in the same way as the total mode energy. The phase of the perturbations in all components is controlled by the dominant mode. The dominant mode is the mode which is driving the system at any given time.

As the global compressional mode is dominating in phase 1, the phase remains constant for all magnetic perturbations. During phase 2 the amplitude of the azimuthal component stays constant because of the lack of available compressional energy. The amplitude of the radial perturbation remains finite because of contributions from the Alfvén mode. The mismatch between the eigenfrequency of the field line (toroidal mode frequency) and the poloidal mode frequency in this discretized system causes its phase to alter gradually. The compressional components display changes in phase during phase 3 , as the toroidal mode is now driving the system and is coupling its energy back into the poloidal mode. In phase 4 the field line resonance is excited anew with a shifted phase.

To confirm the idea that the cycle time is controlled by the phase cycle of the standing waves on the two critical shells closest to the FLR and to learn more 

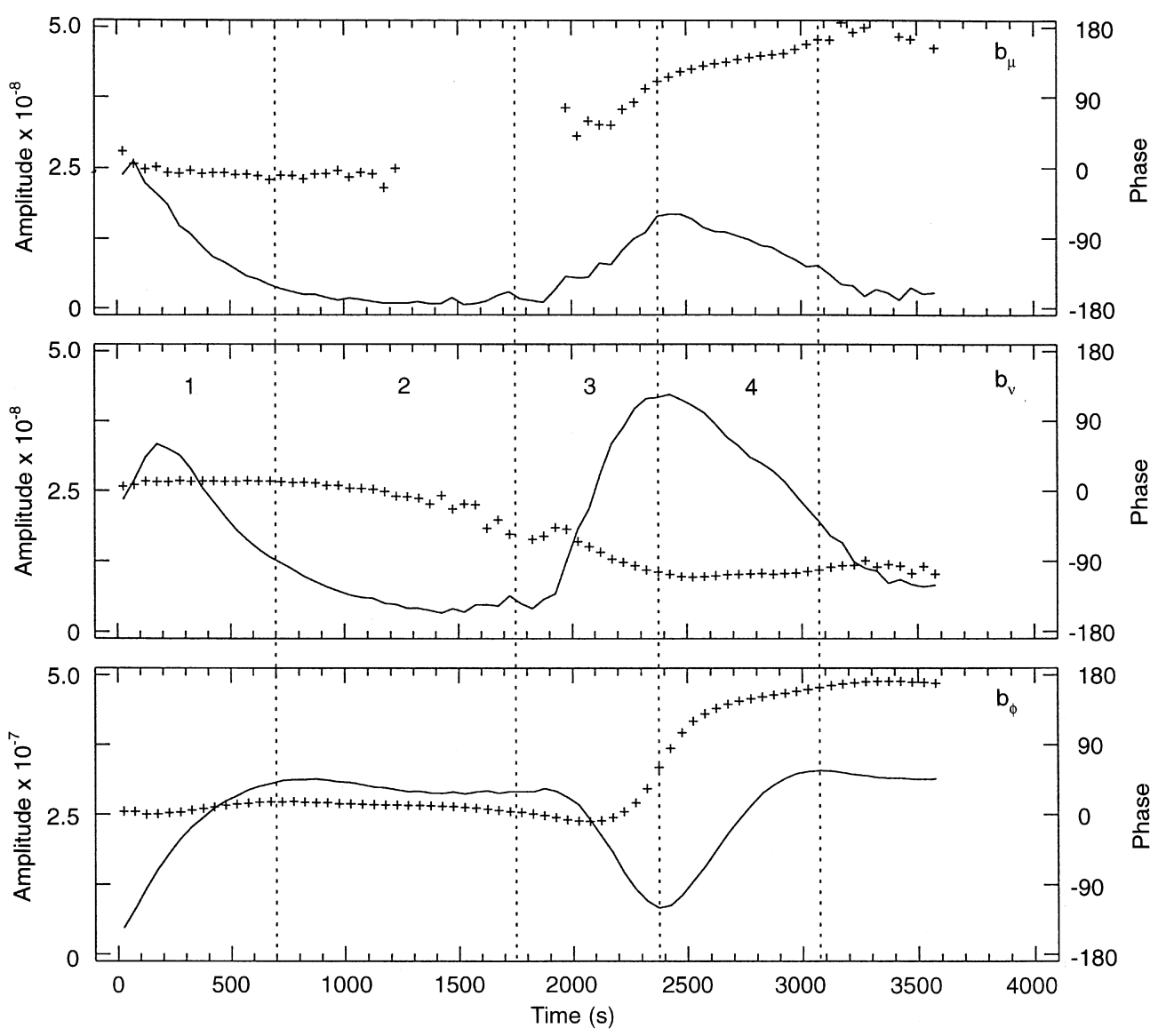

Fig. 5. The temporal development of amplitude (solid line) and phase (symbols) of the magnetic field components for a selected frequency band around $20 \mathrm{mHz}$ that includes the resonant frequency in the system (high resolution grid)

about the relationship between amplitude and phase, a cross-phase analysis was performed for each of the magnetic field components. Time series from those two magnetic shells, again registered near the equatorial plane, were Fourier analyzed. The phase difference $\Delta \Phi_{12}$ between the perturbations was obtained by

$\Delta \Phi_{12}=\operatorname{atan}\left(\frac{R e_{1} I m_{2}-I m_{1} R e_{2}}{R e_{1} R e_{2}+I m_{1} I m_{2}}\right)$

(e.g., Waters et al., 1991). Figure 6 displays $\Delta \Phi_{12}$ in the selected frequency band around $20 \mathrm{mHz}$ for all magnetic components. In $b_{\mu}$ the phase difference remains zero at all times, which is consistent with the model of global compressional eigenoscillations of the magnetosphere.

The phase difference of the perturbations in $b_{v}$ shows influences of both wave modes; its frequency varies with radial distance. Near the resonance it is closely related to the frequencies of the pure Alfvén wave, but further away the compressional mode dominates and the frequency of $b_{v}$ is similar to that of $b_{\mu}$.

A more interesting feature is the phase behavior of $b_{\phi}$. Both field lines are initially driven in the same direction by the source. Because the resonant frequency differs on the two selected shells, $\Delta \Phi_{\phi 12}$ changes linearly in time, as indicated by the solid line in Fig. 6. Before the perturbations of the two field lines come back into phase, the energy conversion sets in and $\Delta \Phi_{\phi 12}$ decreases slowly until the maximum of the energy exchange is reached. When the standing Alfvén waves build up again, the phase difference again grows almost linearly as in the initial cycle. The nonlinear behavior during the energy conversion is produced by the aforementioned phase shifts on both magnetic shells on slightly different time scales.

It is interesting to consider why the amplitude of $b_{\phi}$ decreases as the perturbations return to being in-phase. The behavior can be understood in terms of the perturbation currents given by Eq. (1). Standing Alfvén waves drive field aligned currents, whilst the poloidal mode currents close in circuits perpendicular to $\vec{B}_{0}\left(\nabla_{\perp} \cdot \vec{j}=0\right)$. The field aligned currents

$j_{\|}=\frac{1}{\mu_{0}} \frac{1}{h_{v} h_{\phi}}\left(\frac{\partial b_{\phi}^{*}}{\partial v}-\frac{\partial b_{v}^{*}}{\partial \phi}\right)$

are mainly caused by the gradient of $b_{\phi}^{*}$ in the radial direction, because $\partial b_{\phi}^{*} / \partial v \gg \partial b_{v}^{*} / \partial \phi$ for low azimuthal wave numbers near the resonance. The further out of phase the perturbations on the two field lines next to the FLR are, the larger the gradient $\partial b_{\phi}^{*} / \partial v$. When they are in phase, the gradient is at an minimum. As soon as the phase difference is smaller than $\pi / 2$, the field aligned currents diminish and so does the amplitude of the field line resonance.

In an ideal continuum the FLR corresponds to a delta-function. The radial gradient of $b_{\phi}^{*}$ is always large near the resonance and independent of the phase. In 

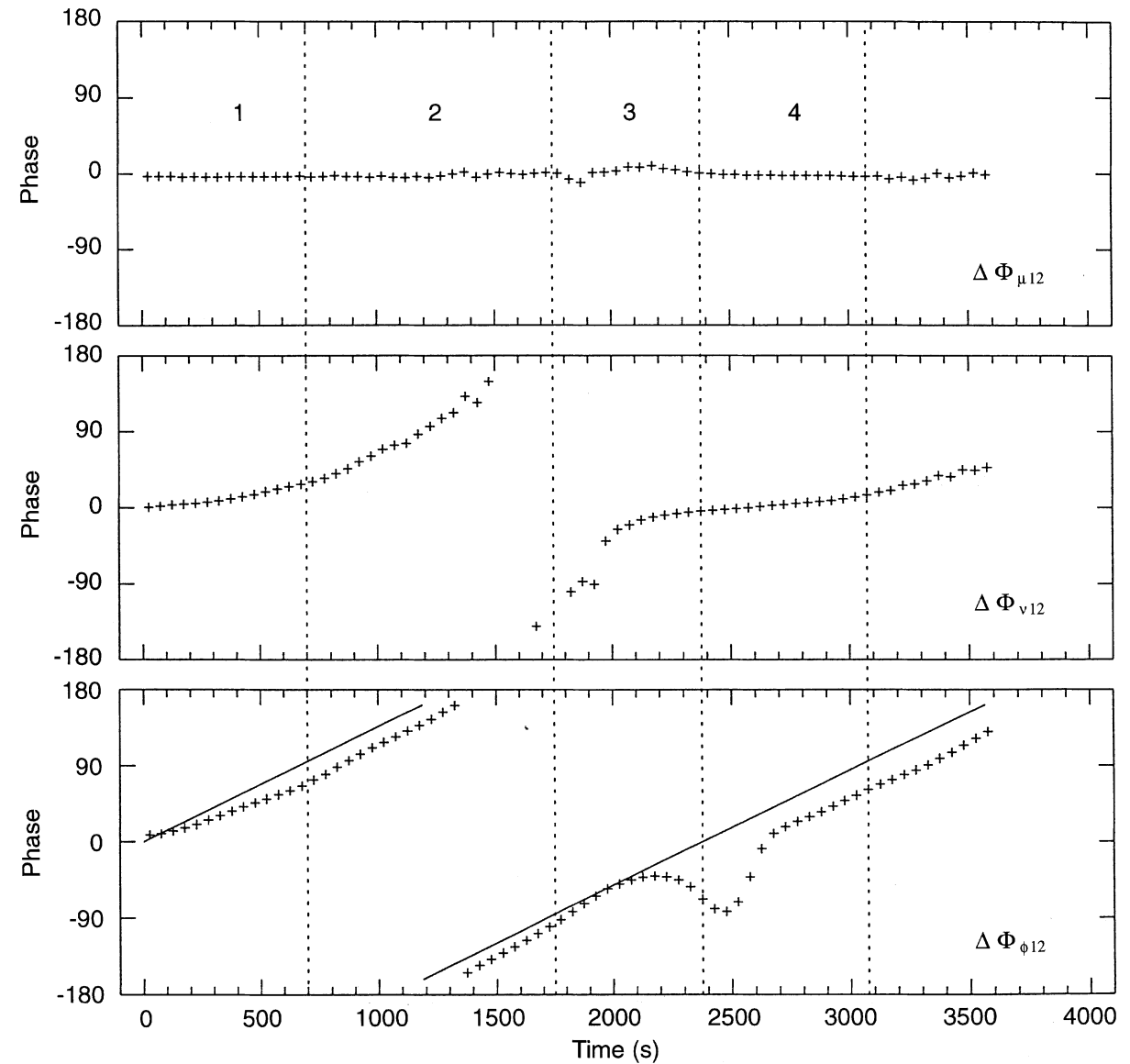

Fig. 6. The temporal development of the phase difference between two magnetic field perturbations (separately for each component) across the FLR for a selected frequency band around $20 \mathrm{mHz}$ that includes the resonant frequency in the system (high resolution grid). The solid line in the lower panel indicates a purely linear phase shift between the two perturbations a discretized system, however, where only samples of the gradient are considered, $\partial b_{\phi}^{*} / \partial v$ depends strongly on the relative phase and can drop to small values.

\section{Possible misinterpretations}

The discretized system produces apparent features that can be linked to observations but are not physically realistic in their origin. An interesting consequence of the discretized model is illustrated in Fig. 7, which shows the magnetic field components at a grid point on the magnetic shell closest to the FLR near the equatorial plane. The simulation in this case was done with the lower resolution grid (41 grid points along $v$ ). The time series displays a few commonly observed features of ULF pulsations. One is wave packeting, which has been observed many times (Waters et al., 1991; Lin et al., 1992; Liao et al., 1994). Possible explanations for wave packeting of observed magnetic (and electric) field amplitudes are, for example, short impulsive energy sources (Waters et al., 1991). Others (Singer et al., 1979) interpret the structures as spatial rather than temporal changes due to the satellite flying through a resonance or a flux tube. However, in the numerical system described here, the wave packeting is produced by the energy conversion in a discretized system. The time scales of the packeting clearly relate to the described mechanism.
Allan et al. (1985) and Allan and Poulter (1989) have shown amplitude modulations near a resonance as a result of beating due to the frequency mismatch between the driving cavity mode and the natural Alfvén frequency on one particular field line. In contrary the interaction presented in this work is caused by the frequency mismatch between two azimuthal perturbations on two neighboring field lines. The beating effect introduced by Allan and coworkers is not visible in these simulations, as the amplitude of the driving cavity mode reduces to small values, compared to the amplitude of the toroidal mode, long before a clear beating effect can occur.

Another feature commonly discussed in connection with observed wave packets is the change of phase of the perturbations between two packets. This type of phase shift is referred to as phase skip. Again several possible explanations have been proposed, such as the presence of multiple, decoupled source mechanisms (Menk and Yumoto, 1994), or one could imagine the existence of multiple spatial structures with distinguishable properties. Again, the phase skips in the time series in Fig. 7 are clearly related to the periodic mode conversion, an artifact produced by the discretization of the continuum.

\section{Assessment and conclusion}

In the last sections some consequences of the discretization of the Alfvén continuum in numerical models were 

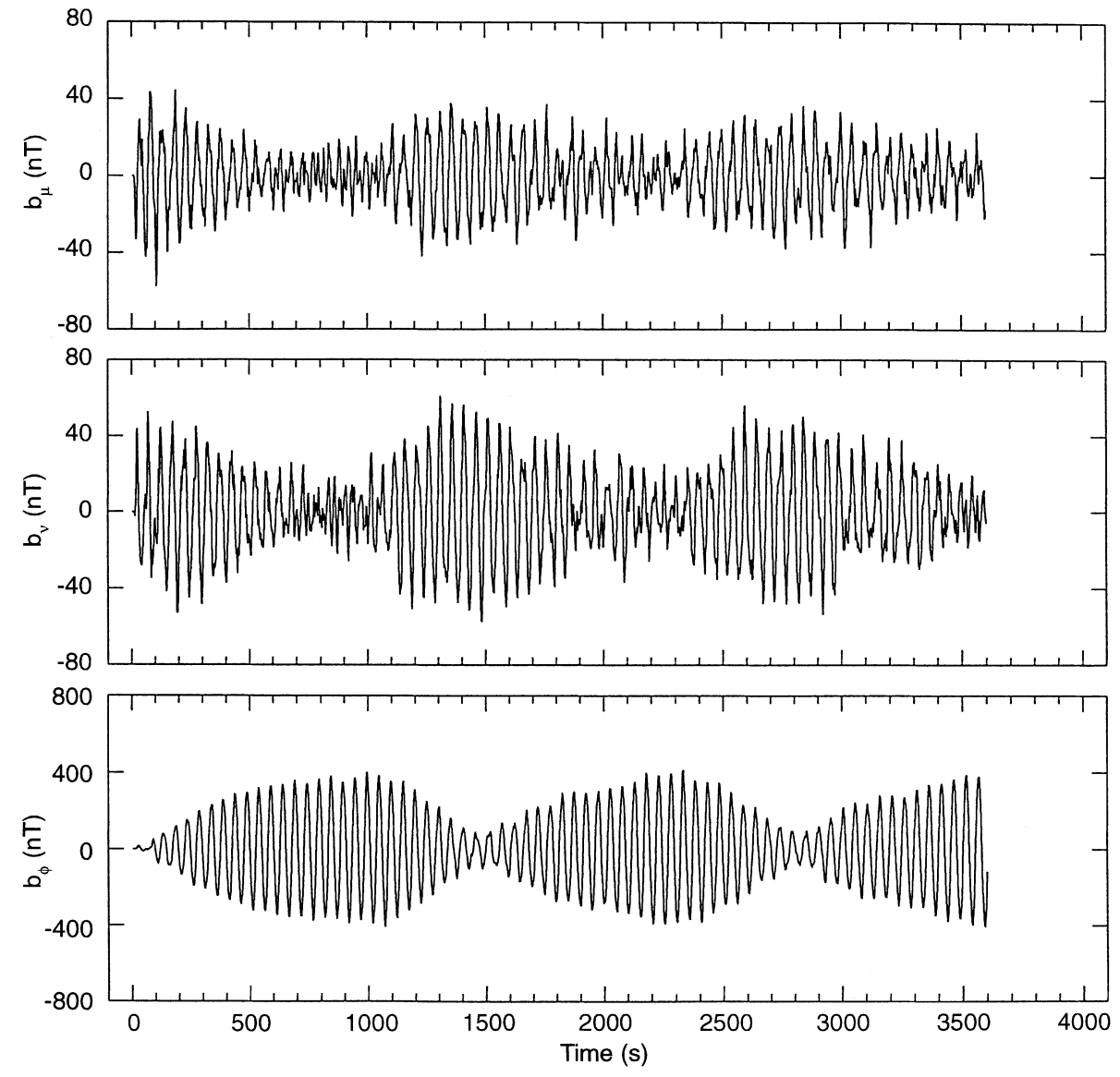

Fig. 7. Time series of the magnetic field components at a grid point near the equatorial plane on the magnetic shell closest to the field line resonance (low resolution grid) illustrated and the important role of the grid dimensions in the radial direction was emphasized. Grid sizes along the field lines and in the azimuthal direction do not control the response of the system. They only have to be selected to satisfy the numerical stability criteria but cause no further problems. The question that naturally arises at this point is: which results from numerical models remain valid?

The numerical model used in this study has produced many useful and commonly accepted results that explain a lot of features connected with ULF pulsations and field line resonances (Lee and Lysak 1989, 1990, 1991a,b). The artifacts shown in the previous section only occurred when the duration of the simulation was extended over many wave cycles. However, the results appear to be realistic within the time limits controlled by frequency difference between azimuthal magnetic field perturbations on two radially neighboring field lines across a dominant FLR. The time limit can be understood as a critical time of the model, and can roughly be estimated by:

$T_{\text {crit }} \approx \frac{3}{4}\left|f_{2}-f_{1}\right|^{-1}$

This corresponds to the time when the azimuthal magnetic perturbations are less than $\pi / 2$ out of phase. The field aligned currents, controlled by the radial gradient of the azimuthal magnetic perturbations across the dominant FLR, begin to diminish and the toroidal mode starts to couple its energy back into the compressional mode. Note that this critical time differs from the time limit governed from the phase mixing criterion (28) by the factor $3 / 4$. The critical time introduced here indicates the beginning of the diversion of the simulation from the true solution.

The analysis discussed so far dealt with a special case in which only one localized field line resonance was important. This is not always the case. In complex systems, such as this three dimensional dipole model, a small change of the background conditions and different forms of initial energy sources can produce a large number of FLRs distributed in radial distance as well as over a wide frequency bandwidth. If there is more than one FLR with considerable amplitude, the artifacts due to the discretization might not appear in investigations of the global energy or other global features. In general the critical time could be governed by the largest frequency difference appearing in the model even if this was between nonresonant field lines. However, the effect of the artifact on the energy conservation in the toroidal mode depends on the amplitude of the transverse oscillation and might be smaller if the nonresonant oscillations had smaller amplitudes.

The fact has to be considered that after the critical time the global compressional mode spreads its energy throughout the model. The problem ceases to be localized. FLRs at many positions absorb energy on 
different time scales, unless the eigenperiod is a linear function of the radial distance. Therefore, even if the system did seem to recover to its original state at a time exceeding $T_{\text {crit }}$, it would have no relevance to the true solution.

Another point to be considered for a discretized system is whether the properties and the behavior of field line resonances are modified, if the actual resonant frequency lies between two grid points. The discrete spectrum of the compressional mode, which is responsible for the excitation of the Alfvén waves, has a finite bandwidth in numerical models as well as in the real magnetosphere, which decreases in time with $t^{-1}$ (Mann et al., 1995). If the eigenfrequencies of the resonant shells lie within this bandwidth, it is valid to represent the coupling to the localized resonances with the model, again provided $t<T_{\text {crit }}$. The exact frequency and position of the nominal maximum of the FLR in numerical models can only be found through a careful investigation of the frequency spectrum of $b_{\mu}$, the purely compressional component that excites the FLR.

The first and most obvious solution to avoid side effects of the discretization of the Alfvén continuum is to simply cut off the simulations before the critical time is reached. An increase of the number of grid points used to represent the continuum in the radial direction would improve the quality of the model during times shorter than $T_{\text {crit }}$ (although it is still a discretization), but the even more important improvement would be that it extends the time limit in which results can be used for a proper interpretation. The consideration of dissipation mechanisms would result in a better representation of the real magnetospheric system, as dissipation broadens the bandwidth of the toroidal spectrum in a natural way and FLRs are resolved more correctly even with only a few grid points. Damping can naturally limit the time over which the excitation is present. A resolution of the grid high enough to guarantee that field line resonances decay in times small relative to $T_{\text {crit }}$ would yield results independent of the discretization.

Acknowledgements. This work was supported by a grant of computer time from the Regionales Rechenzentrum für Niedersachsen, Universität Hannover and financially through a grant by the German Science Foundation and the German Space Agency DARA. Additional support was provided by the Division of Atmospheric Sciences of the National Science Foundation under grant ATM-9314239. R.L. Lysak was supported by the National Science Foundation under grant ATM-9301043.

The Editor in chief thanks F. Menk, W. Allan and S. Poedts for their help in evaluating this paper.

\section{References}

Allan, W., and E. M. Poulter, Damping of magnetospheric cavity modes: A discussion, J. Geophys. Res., 94, 11843-11853, 1989.

Allan, W., and E. M. Poulter, ULF waves - their relationship to the structure of the Earth's magnetosphere, Rep. Prog. Phys., 55, 533-598, 1992.

Allan, W., and D. R. Mc Diarmid, Frequency ratios and resonance positions for magnetospheric cavity/wave guide modes, Ann. Geophysicae, 11, 916-924, 1993.
Allan, W., S. P. White, and E. M. Poulter, Magnetospheric coupling of hydromagnetic waves - initial results, Geophys. Res. Lett., 12, 287-290, 1985.

Allan, W., S. P. White, and E. M. Poulter, Impulse-excited hydromagnetic cavity and field line resonances in the magnetosphere, Planet. Space Sci., 34, 371-385, 1986.

Appert, K., D. Berger, R. Gruber, and J. Rappaz, A new finite element approach to the normal mode analysis in magnetohydrodynamics, J. Comp. Physics, 18, 284-299, 1975.

Appert, K., J. Vaclavik, and L. Villard, Spectrum of low-frequency, nonaxisymmetric oscillations in a cold, current-carrying plasma column, Phys. Fluids, 27, 432-437, 1984.

Barston, E. M., Electrostatic oscillations in inhomogeneous cold plasmas, Ann. Phys. N.Y., 29, 282, 1964.

Bertin, G., G. Einaudi, and F. Pegoraro, Alfvén modes in inhomogeneous plasmas, Comments Plasma Phys. Controlled Fusion, 10, 173-184, 1986.

Chen, L., and A. Hasegawa, A theory of long-period magnetic pulsations, 1. Steady state excitation of field line resonance, J. Geophys. Res., 79, 1024-1032, 1974a.

Chen, L., and A. Hasegawa, A theory of long-period magnetic pulsations, 2. Impulse excitation of surface eigenmode, J. Geophys. Res., 79, 1033-1037, 1974b.

Cummings, W. D., R. J. O'Sullivan, and P. J. Coleman, Jr., Standing Alfvén waves in the magnetosphere, J. Geophys. Res., 74, 778-793, 1969.

Ding, D. Q., R. E. Denton, M. K. Hudson, and R. L. Lysak, An MHD simulations study of the poloidal mode field line resonance in the Earth's dipole magnetosphere, J. Geophys. Res., 100, 63-77, 1995.

Dungey, J. W., Electrodynamics of the outer atmosphere, Penn., State Univ., Ionos. Res. Lab. Sci. Rep. 69, 1954.

Hameiri, E., On the essential spectrum of ideal magnetohydrodynamics, Commun. Pure Appl. Math. 38, 43-66, 1985.

Inhester, B., Resonance absorption of Alfvén oscillations in a nonaxisymmetric magnetosphere, J. Geophys. Res., 91, 1509, 1986.

Inhester, B., Numerical modeling of hydromagnetic wave coupling in the magnetosphere, J. Geophys. Res., 92, 4751-4756, 1987.

Kivelson, M. G., and D. J. Southwood, Resonant ULF waves: A new interpretation, Geophys. Res. Lett., 12, 49-52, 1985.

Kivelson, M. G., and D. J. Southwood, Coupling of global magnetospheric MHD eigenmodes to field line resonances, J. Geophys. Res., 91, 4345-4351, 1986.

Kivelson, M. G., J. Etcheto, and J. G. Trotignon, Global compressional oscillations of the terrestrial magnetosphere: the evidence and a model, J. Geophys. Res., 89, 9851-9856, 1984.

Kouznetsov, I., and W. Lotko, Radial energy transport by magnetospheric ULF waves: effects of magnetic curvature and plasma pressure, J. Geophys. Res., 100, 7599-7612, 1995.

Lee, D.-H., and R. L. Lysak, Magnetospheric ULF wave coupling in the dipole model: the impulsive excitation, J. Geophys. Res., 94, 17097-17103, 1989.

Lee, D.-H., and R. L. Lysak, Effects of azimuthal asymmetry on ULF waves in the dipole magnetosphere, Geophys. Res. Lett., 17, 53-56, 1990.

Lee, D.-H., and R. L. Lysak, Impulsive excitation of ULF waves in the three-dimentional dipole model: the initial results, J. Geophys. Res., 96, 3479-3486, 1991a.

Lee, D.-H., and R. L. Lysak, Monochromatic ULF wave excitation in the dipole magnetosphere, J. Geophys. Res., 96, 5811-5817, $1991 b$.

Lee, M. A., and B. Roberts, On the behaviour of hydromagnetic surface waves, Astrophys. J., 301, 430-439, 1986.

Liao, B., J. R. Benbrook, E. A. Bering III, G. J. Byrne, J. R. Theall, L. J. Lanzerotti, and C. G. Maclennan, Balloon observations of nightside Pc5 quasi-electrostatic waves above the south pole, J. Geophys. Res., 99, 3879-3891, 1994.

Lifshitz, A. E., Continuous spectrum in general toroidal systems (ballooning and Alfvén modes), Phys. Lett. A, 122, 350-356, 1987. 
Lin, N., M. J. Engebretson, L. A. Reinleitner, J. V. Olson, D. L. Gallagher, L. J. Cahill Jr., J. A. Slavin, and A. M. Persoon, Field and thermal plasma observations of ULF pulsations during a magnetically disturbed interval, J. Geophys. Res., 97, 14859-14875, 1992.

Lysak, R. L., and D. H. Lee, Response of the dipole magnetosphere to pressure pulses, Geophys. Res. Lett., 19, 937-940, 1992.

Mann, I. R., and A. N. Wright, Finite lifetimes of ideal poloidal Alfvén waves, J. Geophys. Res., 100, 23677-23686, 1995.

Mann, I. R., A. N. Wright, and P. S. Cally, Coupling of magnetospheric cavity modes to field line resonances: a study of resonance widths, J. Geophys. Res., 100, 19441-19456, 1995.

Menk, F. W., and K. Yumoto, Conjugate and meridional phase structure of low latitude ULF pulsations, Adv. Space Res., 5, 425-428, 1994.

Nishida, A., Geomagnetic diagnosis of the magnetosphere, Physics and Chemistry in Space, vol. 9, Springer, Berlin, Heidelberg New York, 1978.

Parker, E. N., Interaction of solar wind with the geomagnetic field, Phys. Fluids, 1, 171, 1958.

Pritchett, P. L., and J. M. Dawson, Phase mixing in the continuous spectrum of Alfvén waves, Phys. Fluids, 21, 516-518, 1978.

Radoski, H. R., A note on the problem of hydromagnetic resonances in the magnetosphere, Planet. Space Sci., 19, 1012, 1971.

Radoski, H. R., A Theory of latitude dependent geomagnetic micropulsations: the asymptotic fields, J. Geophys. Res., 79, 595-603, 1974.

Radoski, H. R., Hydromagnetic waves: temporal development of coupled modes, Air Force Geophysics Laboratory (PHG), Massachusetts, Rep. AFGL-TR-76-0104, 1976.
Rankin, R., J. C. Samson, and P. Frycz, Simulations of driven field line resonances in the Earth's magnetosphere, J. Geophys. Res., 98, 21341-21352, 1993.

Rickard, G. J., and A. N. Wright, Alfvén resonance excitation and fast wave propagation in magnetospheric waveguides, J. Geophys. Res., 99, 13455-13464, 1994.

Sedlacek, Z., Electrostatic oscillations in cold inhomogeneous plasma, I. Differential equation approach, J. Plasma Phys., 5, 239-263, 1971.

Singer, H. J., C. T. Russell, M. G. Kivelson, T. A. Fritz, and W. Lennartsson, Satellite observations of the spatial extent and structure of $\mathrm{Pc} 3,4,5$ pulsations near the magnetospheric equator, Geophys. Res. Lett., 6, 889-892, 1979.

Southwood, D. J., The hydromagnetic stability of the magnetospheric boundary, Planet. Space Sci., 16, 587, 1968.

Southwood, D. J., Some features of field line resonances in the magnetosphere, Planet. Space Sci., 22, 483-491, 1974.

Southwood, D. J., and M. G. Kivelson, The magnetohydrodynamic response of the magnetospheric cavity to changes in solar wind pressure, J. Geophys. Res., 95, 2301, 1990.

Waters, C. L., F. W. Menk, B. J. Fraser, and P. M. Ostwald, Phase structure of low-latitude Pc3-4 pulsations, Planet. Space Sci., 39, 569-582, 1991.

Wright, A. N., MHD theory of magnetic pulsations, in: Physical signatures of magnetospheric boundary layer processes, Ed. J.A. Holtet and A. Egeland, NATO ASI Series C, vol. 425, 1994.

Wright, A. N., and M. J. Thompson, Analytical treatment of Alfvén resonances and singularities in nonuniform magnetoplasmas, Phys. Plasmas, 1, 691-705, 1994.

Zhu, X. M., and M. G. Kivelson, Analytic formulation and quantitative solutions of the coupled ULF wave problem, $J$. Geophys. Res., 93, 8602-8612, 1988. 